\title{
Prophylactic donor lymphocyte infusion after allogeneic stem cell transplantation in acute leukaemia - a matched pair analysis by the Acute Leukaemia Working Party of EBMT
}

Christoph Schmid, ${ }^{1}$

Myriam Labopin, ${ }^{2}$ Nicolaas Schaap, ${ }^{3}$

Hendrik Veelken, ${ }^{4}$ Michael Schleuning, ${ }^{5}$ Michael Stadler, ${ }^{6}$ Juergen Finke, ${ }^{7}$ Erin Hurst, ${ }^{8}$ Frederic Baron, Olle Ringden, ${ }^{10}$ (D) Gesine Bug, ${ }^{11}$ Didier Blaise, ${ }^{12}$ Johanna Tischer, ${ }^{13}$ Adrian Bloor, ${ }^{14}$ Jordi Esteve, ${ }^{15}$ Sebastian Giebel, ${ }^{16}$ Bipin Savani, ${ }^{17}$ Norbert-Claude Gorin, ${ }^{18}$ Fabio Ciceri, ${ }^{19}$ (iD) Mohamad Mohty, ${ }^{20, *}$ Arnon Nagler ${ }^{21, *}$ and on behalf of the EBMT Acute Leukaemia Working Party ${ }^{1}$ Department of Haematology and Oncology, Klinikum Augsburg, University of Munich, Augsburg, Germany, ${ }^{2}$ Faculté de Médicine SaintAntoine and EBMT data office, Paris, France,

${ }^{3}$ Radboud University Medical Centre, Nijmegen, ${ }^{4}$ BMT Centre Leiden, Leiden University Hospital, Leiden, The Netherlands, ${ }^{5}$ Deutsche Klinik für Diagnostik, KMT Zentrum, Wiesbaden, ${ }^{6}$ Department of Haematology and Oncology, Medizinische Hochschule Hannover, Hannover,

${ }^{7}$ Department of Medicine, Haematology and Oncology, University of Freiburg, Freiburg, Germany, ${ }^{8}$ Bone Marrow Transplant Unit, NCCN

Freeman Hospital, Newcastle-upon-Tyne, United Kingdom, ${ }^{9}$ Department of Medicine, Division of Haematology, University of Liège, Liège, Belgium, ${ }^{10}$ Department of Laboratory Medicine, Division of Therapeutic Immunology, Karolinska Institutet, Karolinska University Hospital Huddinge, Stockholm, Sweden, ${ }^{11}$ Department of Medicine 2, Haematology and Oncology, Universitaetsklinikum, Frankfurt Germany, ${ }^{12}$ Programme de Transplantation \& Therapie Cellulaire-Centre de Recherche en Cancérologie de Marseille-Institut Paoli Calmettes, Marseille, France, ${ }^{13}$ Department of Medicine 3, Haematology and Oncology, Ludwig-Maximilians-University, Munich, Germany, ${ }^{14}$ Stem Cell

Transplantation Unit, The Christie NHS Foundation Trust, Manchester, United Kingdom,

\section{Summary}

Strategies for relapse prevention after allogeneic transplantation in acute leukaemia are warranted. A registry-based matched-pair analysis evaluated the efficacy of prophylactic donor lymphocyte infusion (proDLI). Adults receiving proDLI in complete remission (CR) and controls were pair-matched for age, diagnosis, cytogenetics, stage, donor, gender, conditioning and T-cell depletion. Eighty-nine pairs were identified (median follow-up: 6.9 years). Within the entire cohort, no difference was observed. However, among patients with high-risk acute myeloid leukaemia (AML) (unfavourable cytogenetics and/or transplanted beyond first CR), proDLI recipients had improved overall survival $(69.8 \%$ vs. $40.2 \%$ in controls, $P=0.027)$. ProDLI has moderate efficacy, but can contribute to improved outcome in high-risk AML.

Keywords: acute leukaemia, allogeneic stem cell transplantation, DLI, relapse prevention. 


\author{
${ }^{15}$ Department of Haematology, Hospital Clinic, \\ IDIBAPS, Barcelona, Spain, ${ }^{16}$ Maria Sklo- \\ dowska-Curie Cancer Center and Institute of \\ Oncology, Gliwice Branch, Gliwice, Poland, \\ ${ }^{17}$ Long term Transplant Clinic, Vanderbilt \\ University Medical Center, Nashville, TN, USA, \\ ${ }^{18}$ Faculté de Médicine Saint-Antoine and EBMT \\ data office, Paris, France, ${ }^{19}$ Department of Hae- \\ matology, Osp. San Raffaele, Università degli \\ Studi, Milano, Italy, ${ }^{20}$ Faculté de Médicine \\ Saint-Antoine and EBMT data office, Paris, \\ France and ${ }^{21}$ Chaim Sheba Medical Centre, Tel- \\ Hashomer, Israel
}

Received 8 August 2018; accepted for

publication 15 October 2018

Correspondence: Christoph Schmid,

Department of Haematology and Oncology,

Klinikum Augsburg, University of Munich,

Stenglinstrasse 2, D-86156 Augsburg, Germany.

E-mail: Christoph.Schmid@klinikum-

augsburg.de

* MM and AN contributed equally to this work

and share final co-authorship

Relapse is the most frequent cause of failure after allogeneic stem cell transplantation (SCT) for acute myeloid or lymphoblastic leukaemia (AML/ALL). Therefore, donor lymphocyte infusion (DLI) has been given in complete haematological remission (CHR) for relapse prevention. In most studies, DLI was applied pre-emptively, i.e. triggered by mixed donor chimerism (Krishnamurthy et al, 2013), persisting minimal residual disease (MRD) or molecular relapse (Dominietto et al, 2007; Bejanyan et al, 2015). In contrast, DLI given without any sign of the underlying disease in full chimeras was called prophylactic DLI (proDLI). Several studies reported on improved outcome after proDLI as compared to historical controls (Wang et al, 2012a,b; Eefting et al, 2014; Jedlickova et al, 2016). However, the reported populations and details of proDLI were extremely heterogenous, and no large scale, balanced comparison is available. Hence, a registry-based analysis was performed to evaluate the efficacy of proDLI in acute leukaemia.

\section{Methods}

Adult recipients of proDLI were selected from the European Society for Blood and Marrow Transplantation (EBMT) Acute Leukaemia Working Party (ALWP) registry, based on the following criteria: (i) SCT from matched sibling (MSD) or matched unrelated donors (MUD, 9/10 or 10/10 human leucocyte antigen [HLA] matches) between 2000 and 2011, (ii) CHR post-transplant without $\mathrm{MRD} /$ molecular relapse, (iii) full donor chimerism, (iv) DLI within 1 year from SCT without additional anti-leukaemic or lympho-depleting therapy, (v) no leukaemia relapse between the date of transplant and DLI, (vi) no history of acute graft-versus-host disease (aGvHD) greater than grade I or chronic GvHD (cGvHD) before DLI, and (vii) no active GvHD at the time of DLI. Controls were selected from the same registry and fulfilled inclusion criteria $1-4$, but had not received proDLI, based on the centres' decision.

The efficacy of proDLI was studied using a matched pair analysis among proDLI recipients and controls. Matching factors were: Age at SCT ( \pm 5 years), diagnosis (AML/ALL), cytogenetics (good/intermediate/poor/failed), stage at SCT (first $\mathrm{CR}$ [CR1]/second CR [CR2]/active disease), donor (MSD/ MUD), recipient-donor gender combination, conditioning (reduced/standard), and T-cell depletion of the graft (none/ in vivo/ex vivo). To avoid immortal time bias, controls had to be alive and free of aGvHD grade II-IV, cGvHD and relapse at least as long as the time interval from transplant to proDLI in the respective matched proDLI recipient. The study was performed in accordance with the revised Helsinki declaration, and was approved by the general assembly of the ALWP.

\section{Statistics}

Follow-up was calculated using the reverse Kaplan-Meier method. Overall and leukaemia-free survival (OS/LFS) were estimated using the Kaplan-Meier method. Cumulative incidence functions were used to estimate relapse incidence (RI) and non-relapse mortality (NRM) in a competing risk 
setting. The two cohorts were compared using (paired) Wilcoxon signed rank test for continuous, and McNemar test for categorical variables. Outcomes were compared using the Cox proportional hazard model. To account for the association within matching groups, we introduced a random effect or frailty for each matched pair into the model (Therneau et al, 2003). Potential Interactions between use of proDLI and all other covariates were tested. Results were expressed as the hazard ratio (HR) with $95 \%$ confidence interval (95\% $\mathrm{CI}$ ). All tests were two-sided, type I error rate fixed at 0.05 . R statistical software version 3.2.3 L (https://www.R-project. org/) was used. As planned previously, sub-analyses were stratified by diagnosis (ALL or AML) and risk groups. Highrisk AML was defined by unfavourable cytogenetics and/or SCT not in CR, standard-risk by favourable/intermediate cytogenetics and SCT in CR.

\section{Results}

One hundred and five patients fulfilled the inclusion criteria for the proDLI cohort and 13827 were available as controls. Eighty-nine matched pairs were identified (Table I). Among

\begin{tabular}{|c|c|c|c|}
\hline & Controls & proDLI recipients & Test $P$-value (paired) \\
\hline Number & 89 & 89 & \\
\hline \multicolumn{4}{|l|}{ Diagnosis } \\
\hline AML & $65(73 \%)$ & $65(73 \%)$ & \multirow[t]{2}{*}{1} \\
\hline ALL & $24(27 \%)$ & $24(27 \%)$ & \\
\hline \multicolumn{4}{|c|}{ Patient age at SCT (years) } \\
\hline Median (range) & $44.1(19.4-67.4)$ & $44.3(18.2-69.9)$ & 0.32 \\
\hline \multicolumn{4}{|l|}{ Year of SCT* } \\
\hline median & 2006 & 2007 & 0.16 \\
\hline \multicolumn{4}{|l|}{ Cytogenetic subgroup } \\
\hline Good risk & $8(9 \%)$ & $8(9 \%)$ & \multirow[t]{4}{*}{1} \\
\hline Intermediate risk & $56(63 \%)$ & $56(63 \%)$ & \\
\hline Poor risk & $18(20 \%)$ & $18(20 \%)$ & \\
\hline Failed/missing & $7(8 \%)$ & $7(8 \%)$ & \\
\hline \multicolumn{4}{|l|}{ Stage at SCT } \\
\hline CR1 & $64(72 \%)$ & $64(72 \%)$ & \multirow[t]{3}{*}{1} \\
\hline $\mathrm{CR} 2 / 3$ & $9(19 \%)$ & $9(19 \%)$ & \\
\hline Active disease & $16(18 \%)$ & $16(18 \%)$ & \\
\hline \multicolumn{4}{|l|}{ Donor type } \\
\hline MSD & $55(62 \%)$ & $55(62 \%)$ & \multirow[t]{2}{*}{1} \\
\hline MUD & $34(38 \%)$ & $34(38 \%)$ & \\
\hline \multicolumn{4}{|c|}{ Female donor for male recipient } \\
\hline No & $80(90 \%)$ & $80(90 \%)$ & \multirow[t]{2}{*}{1} \\
\hline Yes & $9(10 \%)$ & $9(10 \%)$ & \\
\hline \multicolumn{4}{|l|}{ Conditioning } \\
\hline Myeloablative & $61(69 \%)$ & $61(68.54 \%)$ & \multirow[t]{2}{*}{1} \\
\hline Reduced & $28(31)$ & $28(31.46 \%)$ & \\
\hline \multicolumn{4}{|l|}{ T-cell depletion } \\
\hline No & $8(9 \%)$ & $8(9 \%)$ & \multirow[t]{3}{*}{1} \\
\hline In vivo & $41(46 \%)$ & $41(46 \%)$ & \\
\hline Ex vivo & $40(45 \%)$ & $40(45 \%)$ & \\
\hline \multicolumn{4}{|l|}{ Stem cell source* } \\
\hline Bone marrow & $13(15 \%)$ & $17(19 \%)$ & \multirow[t]{2}{*}{0.54} \\
\hline Peripheral blood & $76(85 \%)$ & $72(81 \%)$ & \\
\hline \multicolumn{4}{|c|}{ Previous autologous SCT ${ }^{*}$} \\
\hline No & $88(99 \%)$ & $89(100 \%)$ & \multirow[t]{2}{*}{1} \\
\hline Yes & $1(1 \%)$ & $0(0 \%)$ & \\
\hline \multicolumn{4}{|c|}{ Previous allogeneic $\mathrm{SCT}^{\star}$} \\
\hline No & $88(99 \%)$ & $88(99 \%)$ & \multirow[t]{2}{*}{1} \\
\hline Yes & $1(1 \%)$ & $1(1 \%)$ & \\
\hline
\end{tabular}

Table I. Patient characteristics and matching results.

SCT, stem cell transplantation, CHR, complete haematological remission, MSD, matched sibling donor; URD, unrelated donor.

*variable not used for matching. 
proDLI recipients, the median interval from SCT to first proDLI was 163 (42-346) days. Patients received a median of 2 infusions, the median number of CD3+ cells at proDLI1 was $3 \times 10^{6} / \mathrm{kg}\left(1 \times 10^{7} / \mathrm{kg}\right.$ in MSD, $0.5 \times 10^{6} / \mathrm{kg}$ in MUD $)$. Following proDLI, 14 patients (15.7\%) developed aGvHD (grade I/2, $n=10$, grade III/IV, $n=4$ ). The cumulative incidence of aGvHD grade III/IV was $4.5 \%$. Twenty-five patients developed cGvHD (cumulative incidence: $28 \%$; Table SI).

Median follow-up was 9.2 (range: 1;9-12;6) and 7.3 (range: 0.5-15) years for proDLI recipients and controls. When comparing the outcome of proDLI recipients and controls, no difference was detected with respect to any endpoint (Table SI). Higher CD3+ counts at proDLI1 were associated with non-significant trend for reduced RI and higher NRM, resulting in identical survival. Similarly, outcome was not influenced by the occurrence of cGvHD following proDLI.

In the pre-planned subgroup analysis, no advantage of proDLI was found in standard-risk AML and ALL (Table SII). In contrast, in high-risk AML, proDLI recipients had a significantly improved 5-year OS (69.8\% vs. $40.2 \%$ among controls; $P=0.027, \quad \mathrm{HR}=0.387 \quad$ (95\% CI: $0.116-$ 0.898), Fig 1A-D, Table SI). Even in this subgroup, matching was sufficient among proDLI recipients and controls (Table SIII). The survival advantage was based on a reduced RI (30.5\% after proDLI vs. $46 \%$ among controls, $P=0.20$ ), and a lower NRM rate among proDLI recipients $(7.4 \%$ vs. $18.6 \%$ among controls, $P=0.22)$. There was a non-significant trend towards more $\mathrm{cGvHD}$ among proDLI recipients.

\section{Discussion}

The efficacy of proDLI in acute leukaemia was evaluated by a registry-based matched-pair analysis. While no influence could be shown in ALL and standard-risk AML, patients with high-risk AML showed an improved OS after proDLI.

A beneficial effect of proDLI has been reported by several groups. Wang et al (2012a,b) observed encouraging results of granulocyte colony-stimulating factor (G-CSF)-primed proDLI, followed by short-term immunosuppression, after HLAidentical and haplo-identical SCT for AML. Eefting et al (2014) reported promising data for delayed proDLI after T-cell depleted (TCD) SCT for ALL. A German study investigated proDLI after sequential conditioning in high-risk AML and myelodysplastic syndrome (Jedlickova et al, 2016). Seven-year OS was $67 \%$ after proDLI and $31 \%$ among controls $(P<0.001)$. However, a selection bias and imbalances between the two cohorts could not be ruled out. In our analysis, we aimed to circumvent this shortcoming by the comparison of proDLI recipients to an extensively matched control group. Also, our data are mature, as indicated by a follow-up $>5$ years. Thus, the results represent the best evidence so far for a role of proDLI in high-risk AML.

Nevertheless, our data also suggest that the overall efficacy of unmanipulated proDLI in acute leukaemia is moderate at best, at least if given as reported here, and improved strategies are required for relapse prevention. As relapse frequently occurs during the first months after transplant, earlier application of donor cells might be more effective, although the risk of GvHD is, inevitably, higher.(Reddy et al, 2005) The study reported by Wang et al (2012a), who administered G-CSF-mobilized proDLI by day $+40-60$, followed by short-term GvHD prophylaxis, might show a way to overcome the risk of earlier interventions. Further approaches (summarized in (Lee et al, 2018) included specific cytotoxic $\mathrm{T}$ cells, ex vivo or in vivo stimulation of donor cells, or the combination of checkpoint inhibitors and DLI. In ALL, bispecific antibodies or chimeric antigen receptor-T cells are options for relapse prevention. Modern drugs such as 5-azacytidine, panobinostat or FLT3 inhibitors have also shown efficacy as maintenance therapy in AML. Given that ex vivo studies suggest a synergism of these drugs and immune effector cells (Goodyear et al, 2010; West \& Johnstone, 2014; Mathew et al, 2018), the combination with proDLI, as currently studied by several groups, seems promising.

The safety of proDLI was acceptable. The low incidence of GvHD was possibly due to the long interval between SCT and proDLI1, low initial cell doses in the unrelated/mismatched donor setting, and the exclusion of patients with a history of aGvHD > grade I. Further, a positive effect of proDLI on immune reconstitution has been discussed.(Bellucci et al, 2002) Patient numbers in our study were too small to define the optimal schedule or cell dose of proDLI, however, safety data confirm strategies described earlier (Eefting et al, 2014; Jedlickova et al, 2016).

Our study has some limitations. First, there was no central review of chimerism and MRD. Inclusion was based on individual centre reports, bearing a bias risk due to different techniques and detection levels. Nevertheless, as the study was part of a larger project also evaluating pre-emptive DLI (Schmid et al, 2015), patients with mixed chimerism and measurable MRD according to the individual centres' criteria could be reliably excluded. Second, the efficacy of proDLI might be different when given after reduced-intensity conditioned or TCD transplant, as compared to standard conditioning. We addressed this by inclusion of both conditioning and T-cell depletion into the matching criteria. Nevertheless, given that $>90 \%$ of patients had received some form of TCD, the results might be less applicable to a non-TCD setting. Third, cytogenetics was the only variable reflecting the leukaemia biology that could be included into the matching criteria. We recognize missing information on molecular markers as a typical drawback of a registry-based analysis. However, the relevance of molecular markers in this context is unclear, and any stratification would have been arbitrary. Forth, proDLI were given relatively late after SCT. Thereby, patients and controls might represent a positive selection, in which the efficacy of any adjuvant intervention would be weaker than among patients at higher risk of relapse. This might explain why a proDLI effect could only be demonstrated within the high-risk subgroup. Finally, although using 
(A)

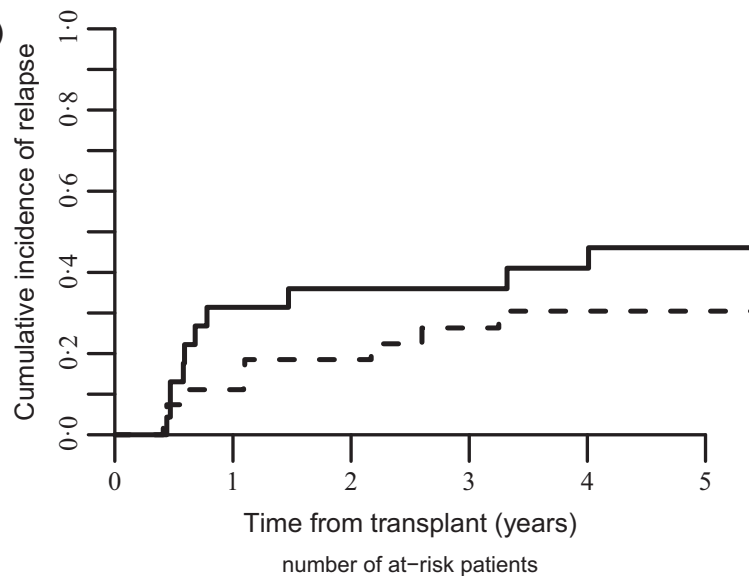

$\begin{array}{lllllll}\text { No proDLI } & 23 & 13 & 11 & 9 & 8 & 5\end{array}$

$\begin{array}{lllllll}\text { proDLI } & 27 & 22 & 19 & 16 & 13 & 13\end{array}$

(C)

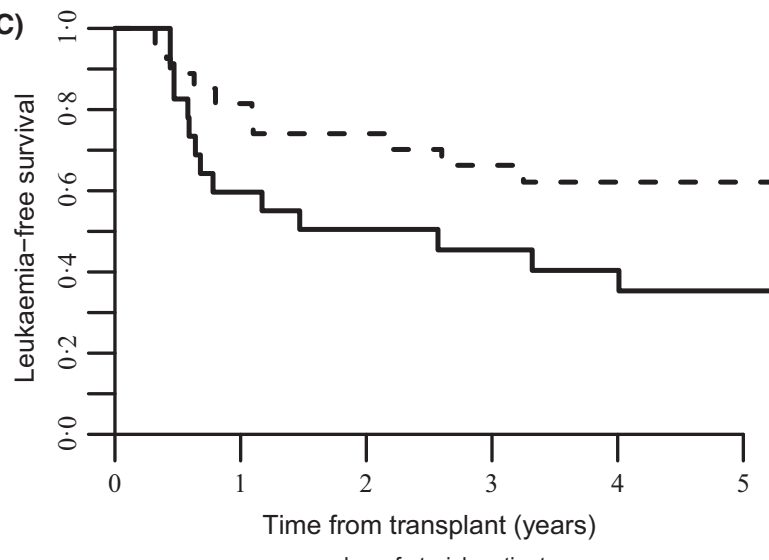

(C)

number of at-risk patients

$\begin{array}{lllcccc}\text { No proDLI } & 23 & 13 & 11 & 9 & 8 & 5 \\ \text { proDLI } & 27 & 22 & 19 & 16 & 13 & 13\end{array}$

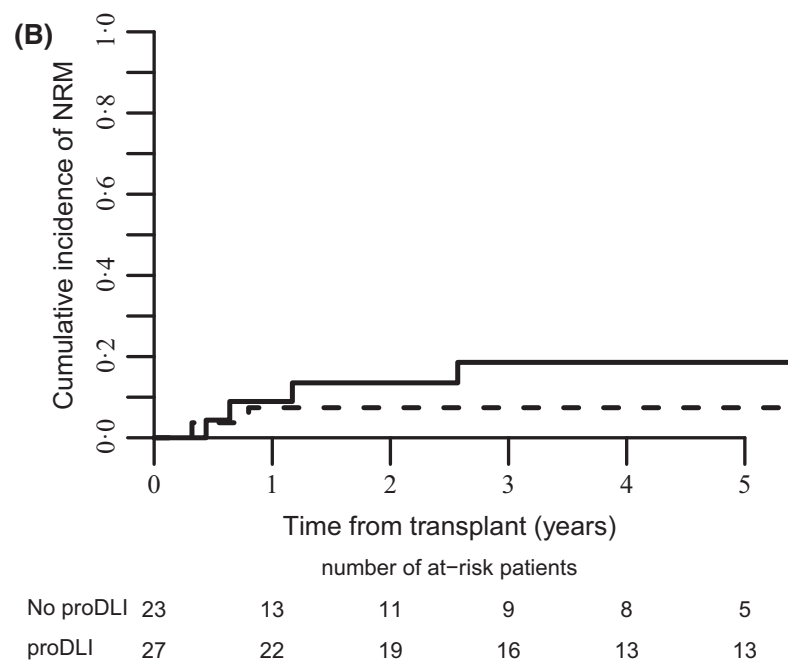

(D)

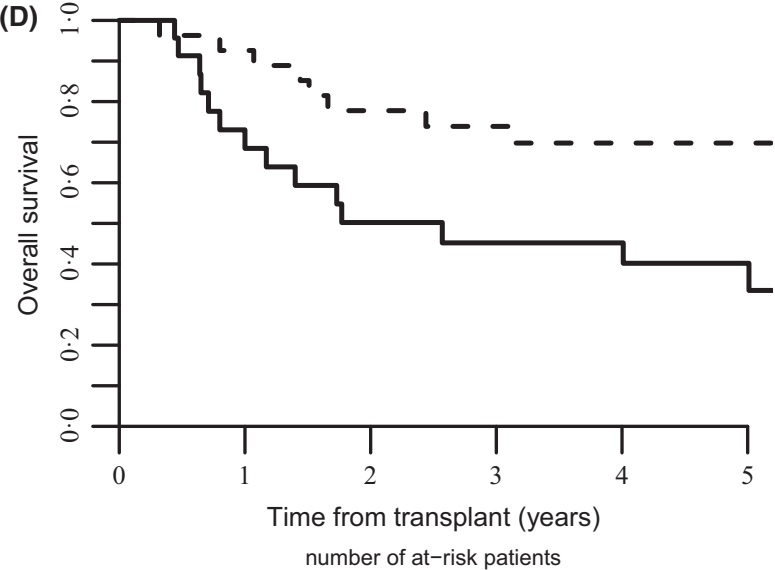

$\begin{array}{llccccc}\text { No proDLI } & 23 & 16 & 11 & 9 & 9 & 6 \\ \text { proDLI } & 27 & 25 & 20 & 18 & 15 & 15\end{array}$

Fig 1. Five-year outcome of pair-matched cohorts who did or did not receive prophylactic DLI (proDLI) in complete haematological remission Subgroup analysis among patients with high-risk acute myeloid leukaemia. (A) Cumulative incidence of relapse (controls: $46.1 \%$ [95\% CI: $23.8-$ 65.9], proDLI recipients: $30.5 \%$ [95\% CI: 14.1-48.7] (B) Cumulative incidence of non-relapse mortality (NRM) (controls: 18.6\% [95\% CI: 5.5-37.8], proDLI recipients: 7.4\% [95\% CI: 1.2-21.4] (C) Leukaemiafree survival (controls: 35.3\% [95\% CI: 14.9-55.8], proDLI recipients: 62.1\% [95\% CI: 43.5-80.7] (D) Overall survival (controls: 40.2\% [95\% CI: 19.3-61], proDLI recipients: 69.8\% [95\% CI: 52.2-87.3].

one of the largest transplant registries worldwide, the numbers remained small. This was due to the strict inclusion criteria, requiring complete information on MRD, chimerism and all variables used for matching. However, considering the many different factors influencing outcome after SCT, such a strictly defined approach seemed mandatory. Certainly, confirmation in a larger cohort or a prospective trial is warranted.

\section{Acknowledgment}

$\mathrm{CS}, \mathrm{ML}, \mathrm{MM}$ and AN designed the study and wrote the manuscript; FB, JE, SG, BS, CG and FC critically reviewed the concept; ML performed the statistical analysis; NS, HV, MS, JF, EH, FB, OR, GB, DB, JT, AB, JE, SG, BS, CG and
FC provided cases for the study. All authors reviewed, edited and finally approved the manuscript. Following EBMT publication rules, co-authorship was offered to centres contributing the highest number of patients. Further, the authors highly appreciate the contribution by many physicians and data managers throughout the EBMT, who made this analysis possible. A list of all contributing centres and responsible physicians is provided in Table SIV. Further, the authors thank Emmanuelle Polge from the ALWP office of the EBMT for data collection.

\section{Supporting Information}

Additional supporting information may be found online in the Supporting Information section at the end of the article. 
Table SI. Outcome of proDLI recipients and controls at 5 years - entire cohort and high-risk AML subgroup.

Table SII. Outcome of proDLI recipients and controls in other subgroups at 5 years.
Table SIII. Patient characteristics and matching results in high-risk AML.

Table SIV. List of participating centers.

\section{References}

Bejanyan, N., Weisdorf, D.J., Logan, B.R., Wang, H.L., Devine, S.M., de Lima, M., Bunjes, D.W. \& Zhang, M.J. (2015) Survival of patients with acute myeloid leukemia relapsing after allogeneic hematopoietic cell transplantation: a center for international blood and marrow transplant research study. Biology of Blood and Marrow Transplantation, 21, 454-459.

Bellucci, R., Alyea, E.P., Weller, E., Chillemi, A., Hochberg, E., Wu, C.J., Canning, C., Schlossman, R., Soiffer, R.J., Anderson, K.C. \& Ritz, J. (2002) Immunologic effects of prophylactic donor lymphocyte infusion after allogeneic marrow transplantation for multiple myeloma. Blood, 99, 4610-4617.

Dominietto, A., Pozzi, S., Miglino, M., Albarracin, F., Piaggio, G., Bertolotti, F., Grasso, R., Zupo, S., Raiola, A.M., Gobbi, M., Frassoni, F. \& Bacigalupo, A. (2007) Donor lymphocyte infusions for the treatment of minimal residual disease in acute leukemia. Blood, 109, 5063-5064.

Eefting, M., Halkes, C.J., de Wreede, L.C., van Pelt, C.M., Kersting, S., Marijt, E.W., von dem Borne, P.A., Willemze, R., Veelken, H. \& Falkenburg, J.H. (2014) Myeloablative T cell-depleted alloSCT with early sequential prophylactic donor lymphocyte infusion is an efficient and safe post-remission treatment for adult ALL. Bone Marrow Transplantation, 49, 287-291.

Goodyear, O., Agathanggelou, A., Novitzky-Basso, I., Siddique, S., McSkeane, T., Ryan, G., Vyas, P., Cavenagh, J., Stankovic, T., Moss, P. \& Craddock, C. (2010) Induction of a CD8 + T-cell response to the MAGE cancer testis antigen by combined treatment with azacitidine and sodium valproate in patients with acute myeloid leukemia and myelodysplasia. Blood, 116, 1908-1918.

Jedlickova, Z., Schmid, C., Koenecke, C., Hertenstein, B., Baurmann, H., Schwerdtfeger, R., Tischer, J., Kolb, H.J. \& Schleuning, M. (2016) Long-term results of adjuvant donor lymphocyte transfusion in AML after allogeneic stem cell transplantation. Bone Marrow Transplantation, 51, 663-667.

Krishnamurthy, P., Potter, V.T., Barber, L.D., Kulasekararaj, A.G., Lim, Z.Y., Pearce, R.M., de Lavallade, H., Kenyon, M., Ireland, R.M., Marsh,
J.C., Devereux, S., Pagliuca, A. \& Mufti, G.J. (2013) Outcome of donor lymphocyte infusion after $\mathrm{T}$ cell-depleted allogeneic hematopoietic stem cell transplantation for acute myelogenous leukemia and myelodysplastic syndromes. Biology of Blood and Marrow Transplantation, 19, $562-568$.

Lee, C.J., Savani, B.N., Mohty, M., Gorin, N.C., Labopin, M., Ruggeri, A., Schmid, C., Baron, F., Esteve, J., Giebel, S., Ciceri, F. \& Nagler, A. (2018) Post-remission strategies for the prevention of relapse following allogeneic hematopoietic cell transplantation for high-risk acute myeloid leukemia: expert review from the Acute Leukemia Working Party of the European Society for Blood and Marrow Transplantation. Bone Marrow Transplantation. [Epub ahead of print]. https://doi.org/10.1038/s41409018-0286-2.

Mathew, N.R., Baumgartner, F., Braun, L., O'Sullivan, D., Thomas, S., Waterhouse, M., Müller, T.A., Hanke, K., Taromi, S., Apostolova, P., Illert, A.L., Melchinger, W., Duquesne, S., Schmitt-Graeff, A., Osswald, L., Yan, K.L., Weber, A., Tugues, S., Spath, S., Pfeifer, D., Follo, M., Claus, R., Lübbert, M., Rummelt, C., Bertz, H., Wäsch, R., Haag, J., Schmidts, A., Schultheiss, M., Bettinger, D., Thimme, R., Ullrich, E., Tanriver, Y., Vuong, G.L., Arnold, R., Hemmati, P., Wolf, D., Ditschkowski, M., Jilg, C., Wilhelm, K., Leiber, C., Gerull, S., Halter, J., Lengerke, C., Pabst, T., Schroeder, T., Kobbe, G., Rösler, W., Doostkam, S., Meckel, S., Stabla, K., Metzelder, S.K., Halbach, S., Brummer, T., Hu, Z., Dengjel, J., Hackanson, B., Schmid, C., Holtick, U., Scheid, C., Spyridonidis, A., Stölzel, F., Ordemann, R., Müller, L.P., Sicre-de-Fontbrune, F., Ihorst, G., Kuball, J., Ehlert, J.E., Feger, D., Wagner, E.M., Cahn, J.Y., Schnell, J., Kuchenbauer, F., Bunjes, D., Chakraverty, R., Richardson, S., Gill, S., Kröger, N., Ayuk, F., Vago, L., Ciceri, F., Müller, A.M., Kondo, T., Teshima, T., Klaeger, S., Kuster, B., Kim, D.D.H., Weisdorf, D., van der Velden, W., Dörfel, D., Bethge, W., Hilgendorf, I., Hochhaus, A., Andrieux, G., Börries, M., Busch, H., Magenau, J., Reddy, P., Labopin, M., Antin, J.H., Henden, A.S., Hill, G.R., Kennedy, G.A., Bar, M., Sarma, A., McLornan, D., Mufti, G., Oran, B., Rezvani, K., Shah, O., Shah, O., Negrin, R.S.,
Nagler, A., Prinz, M., Burchert, A., Neubauer, A., Beelen, D., Mackensen, A., von Bubnoff, N., Herr, W., Becher, B., Socié, G., Caligiuri, M.A., Ruggiero, E., Bonini, C., Häcker, G., Duyster, J., Finke, J., Pearce, E., Blazar, B.R. \& Zeiser, R. (2018) Sorafenib promotes graft-versus-leukemia activity in mice and humans through IL-15 production in FLT3-ITD-mutant leukemia cells. Nature Medicine, 24, 282-291.

Reddy, P., Maeda, Y., Liu, C., Krijanovski, O.I., Korngold, R. \& Ferrara, J.L. (2005) A crucial role for antigen-presenting cells and alloantigen expression in graft-versus-leukemia responses. Nature Medicine, 11, 1244-1249.

Schmid, C., Labopin, L., Veelken, H., Schaap, N., Schleuning, M., Stadler, M.J., Finke, J., Beguin, Y., Collin, M., Ljungman, P., Bug, G., Blaise, D., Tischer, J., Bloor, A., Afanasyev, B., Giebel, S., Gorin, N., Esteve, J., Ciceri, F., Savani, B., Baronn, F., Mailhol, A., Nagler, A. \& Mohty, M. (2015) Efficacy, safety and long term results of prophylactic and preemptive donor lymphocyte infusion after allogeneic stem cell transplantation for acute leukemia: a registry-based evaluation on 343 patients by the acute leukemia working party of EBMT. Blood, 126, 863.

Therneau, T., Grambsch, P. \& Pankratz, V.S. (2003) Penalized survival models and frailty. Journal of Computational and Graphical Statistics, 12, 156-175.

Wang, Y., Liu, D.H., Fan, Z.P., Sun, J., Wu, X.J., Ma, X., Xu, L.P., Liu, K.Y., Liu, Q.F., Wu, D.P. \& Huang, X.J. (2012a) Prevention of relapse using DLI can increase survival following HLAidentical transplantation in patients with advanced-stage acute leukemia: a multi-center study. Clinical Transplantation, 26, 635-643.

Wang, Y., Liu, D.H., Xu, L.P., Liu, K.Y., Chen, H., Zhang, X.H., Chen, Y.H., Han, W., Wang, F.R., Wang, J.Z., Yan, C.H. \& Huang, X.J. (2012b) Prevention of relapse using granulocyte CSFprimed PBPCs following HLA-mismatched/haploidentical, T-cell-replete hematopoietic SCT in patients with advanced-stage acute leukemia: a retrospective risk-factor analysis. Bone Marrow Transplantation, 47, 1099-1040.

West, A.C. \& Johnstone, R.W. (2014) New and emerging HDAC inhibitors for cancer treatment. The Journal of Clinical Investigation, 124, 30-39. 